\title{
The Usefulness of the Recommendations Regarding the Information System Development Method Selection during the Era of Digitalization
}

\author{
Dahlberg, Tomi \\ Turku School of Economics at the \\ University of Turku \\ Turku, Finland \\ tomi.dahlberg@utu.fi
}

\author{
Lagstedt, Altti \\ Turku School of Economics at the \\ University of Turku, \\ Haaga-Helia University of Applied \\ Sciences, Helsinki, Finland \\ altti.lagstedt@haaga-helia.fi
}

\begin{abstract}
The business criticality of information systems (IS) and their development (ISD) appear to have increased recently. Backsourcing, cosourcing and multisourcing of ISD are some of the consequences. They, in turn, extend the need for understanding how to select information systems development methods (ISDM). In this research, we first condensed the knowledge base of ISDM selection research into nine recommendations. We then interviewed 28 ISDM experts and asked them to evaluate how useful the extant ISDM selection recommendations of prior research are to IS user organizations. We discovered that most recommendations were perceived outdated and only limitedly useful. We finally contemplated that paying more attention to how ISDMs are used in business development contexts is a means to increase the usefulness of ISDM selection recommendations.
\end{abstract}

\section{Introduction}

The purpose of this article is to investigate how useful the extant recommendations of information systems development method (ISDM) selection research are for IS user organizations in the selection of ISDMs. Since the 28 interviewed ISDM experts perceived the usefulness of recommendations low, our purpose is also to ponder how to improve the usefulness of ISDM selection recommendations.

The evolution of ISD work and ISDMs during the last 10-15 years motivates our study. Numerous new, especially change-driven (agile), ISDMs have been introduced. The existing ISDM selection models were largely developed prior this era. For example, the probably best known ISDM selection model by Boehm and Turner, called the "Agile and Plan-driven Method
Home Ground -Chart", was published in 2004, and condensed a couple of decades' research [10]. During the last 10-15 years, also two business issues appear to have impacted ISD work and ISDM selection. In IS user organizations, the business criticality of IS, ISD and ISDM (selection) has increased. Several organizations have responded by considering and/or even by executing ISD backsourcing. With ISD backsourcing, we mean actions by which an organization takes at least once outsourced ISD work back into the organization. Secondly, ISD work and hence also ISDM selections and their use appear to have become amalgamated parts of business development, and hence they need to be integrated to business development practices and methods.

During the 1990s and 2000s, the norm was to outsource ISD. IS research provided theoretical and empirical evidence for this [e.g. 4, 32]. The transaction cost economics theory and resource-based views explained that ISD outsourcing offers potential to lower transaction costs and to achieve other business benefits, when the strategic and competitive significance of in-house ISD is low and there are wellfunctioning markets. Digitalization has changed the strategic and competitive significance of ISs. Focus is now on IS and applications that enable and support the development, delivery and operations of an organization's products and services, or even are the products and services. This goes on in digital but also in previously non IS/digital-intensive industries [11]. The business criticality of ISD is in a way reborn in IS user organizations $[7,11,21]$. For ISD the depicted changes mean, among other things, that organizations consider the backsourcing of ISD activities as they seek new balances between outsourced and in-house ISD [7]. Prior research suggests two main reasons for the backsourcing considerations. Firstly, some organizations have been disappointed with the outcomes of ISD outsourcing [7, 39]. Secondly, the era 
of digitalization has profoundly changed the business environments of organizations, that is, the business criticality of digital data and ISs. Organizations respond to these challenges by enhancing their business strategies and, as a part of that, by rethinking their IS sourcing strategies [7, 11, 21].

While considering backsourcing, an organization may need to update its ISD and ISDM knowledge, as well [29]. In line with other prior studies [44], our study [33] showed that IS user organizations tend to abandon their ISD knowledge after ISD is outsourced and/or that, over time, their ISD knowledge becomes outdated. The rapid proliferation of change-driven (agile) ISDMs has characterized the changes in ISD work during the recent years [34, 44]. Still, prior research indicates that no single ISDM suits to the diversity of all ISD projects $[12,15,24]$. One conclusion is that the ISDM selection, the topic of the present article, needs to happen at the ISD project level, case by case [17]. In summary, if an organization needs to update its ISD and ISDM competencies, this should also include the ISDM selection competence.

We investigated ISDM selection in situations where both IS user organizations and IS suppliers participate actively into ISD work. In our opinion, ISD, ISDM and ISDM selection competences are indispensable to IS user organizations in these situations since their objectives for ISD work and projects may differ from those of their IS suppliers [51, 57]. IS suppliers envision ISD projects as their business. They evaluate the success of an ISD project with traditional IS project performance metrics: time, money and the deliverables [26]. That is understandable since IS suppliers are able to influence (only) those metrics with their own actions. They perceive the high values of performance metrics as means to generate more business. For these reasons, an IS supplier may promote the selection and use of ISDM(s) that the supplier understands and masters well. Contrary to this, an IS user organization evaluates the outcomes of an ISD project primarily from business benefits perspective, that is, as means to increase value to customers, internal efficiency, profitability and future competitiveness [26, 45]. Project performance metrics are important but secondary to, or a part of, business benefits. Moreover, the measurement of business benefits is possible only some time after an ISD project has been completed $[26,44,45]$. In summary, we conclude that the ISDM selection recommendations of IS suppliers are not automatically in the interest of IS user organizations. IS user organizations should select the ISDM(s) for an ISD project from business benefits realization and ISD project performance perspectives.

Against the above described backdrop, we decided to investigate whether or not the extant recommendations of ISDM selection research are still useful to IS user organizations that participate actively to ISD with their IS suppliers or consider ISD backsourcing. We saw a research gap here. To fill this gap, we conducted a systematic literature research that included over 1000 unique scientific reports and interviewed 28 ISDM experts. For this research, we defined the following more specific research questions:

RQ1: What are the recommendations of ISDM selection studies and do they include recommendations related to the business development context of ISDMs?

$R Q 2:$ Do the interviewed ISD experts perceive the recommendations of ISDM selection studies useful?

The rest of the article is organized as follows: Next we review ISDM selection research and show how we condensed their ISDM selection criteria into nine recommendations. Section three discusses methodology used in the ISD expert interviews and in the analysis of their responses. The results of the study are disclosed in Section four and we end the article with a discussion and conclusions Section. We especially discuss could better matching between ISDMs and business development contexts provide more useful recommendations. We contribute to research by condensing the criteria of ISDM selection research into well-defined recommendations, by probing their perceived usefulness empirically, and by suggesting how to improve such recommendations.

\section{Theoretical Background}

\subsection{From ISD Outsourcing to Backsourcing}

An IS user organization considers outsourcing, insourcing, backsourcing, cosourcing and multisourcing as its ISD sourcing alternatives [7, 28, 35]. We define these terms in the context of ISD as follows:

- Outsourcing: an IS user organization mandates an IS supplier to develop an IS for the organization

- Insourcing: an IS user organizations executes the development of an IS inside of the organization.

- Backsourcing: an IS user organization takes back at least once outsourced ISD work from IS supplier(s) (partly or wholly) to develop an IS.

- Cosourcing: an IS user organization and an IS supplier collaborate closely to develop an IS.

- Multisourcing: an IS user organization and several IS suppliers collaborate closely to develop an IS.

An IS user organization needs ISDM selection competence in the latter four alternatives. We regard cosourcing and multisourcing as specific forms of backsourcing, and we investigate the usefulness of ISDM selection recommendations in situations where both IS user organizations and IS suppliers collaborate 
closely. Attempts to solve the perceived challenges of ISD outsourcing [7, 20, 28, 35] increased business criticality of IS $[7,11]$ and the proliferation of new ISDMs were discussed above as the motives behind backsourcing. ISD outsourcing has rigid theoretical and practical knowledge basis [e.g. 7, 32] to save costs, to reduce ISD risks and to free resources to core business [20, 46, 57]. Empirical research has, however, produced mixed results [e.g. 4, 41]. For some time ISD outsourcing was a hype term and a management fashion $[1,35]$, which led some organizations to place unrealistic expectations. Quality and cost problems [7, $20,35]$, as well as inflexibilities in reactions to IS user organization's changing business needs [7] have been reported as typical ISD outsourcing challenges. Partial or full backsourcing is one of the means to remedy past miscalculations [7, 20, 35]. Moreover, backsourcing is the preferable alternative according to the theoretical basis of (out)sourcing if the role of ISD transforms and becomes business critical and/or a part of the core business in an organization $[7,11]$.

Above we reasoned that IS suppliers' ISDM selection recommendations may differ from the interests of IS user organizations. This conclusion deserves additional elaboration. It seems obvious that IS user organizations with insourced ISD have, at the minimum, some ISDM and ISDM selection competences. Similar competences are needed in backsourcing, as well, since ISD is carried out at least for the execution period of an ISD project $[28,29]$. In cosourcing and multisourcing, IS suppliers are typically responsible for the operative-level coding and implementation of software. IS user organizations are responsible for business and use cases/requirements, user testing and business (process) development. IS user organizations also bear the accountability for the success of ISD projects. Due to their accountabilities, IS user organizations need to understand that the pros and cons of various ISDMs are suitable to their ISD projects [29]. Based on such understanding, an IS user organization is able to select the most suitable ISDM for an ISD project and the most suitable IS supplier to implement the project. Some IS suppliers are unwilling to use ISDMs unfamiliar to them even if those ISDMs are widely used by other suppliers [33]. It appears risky to allow an IS supplier to select the ISDM alone for ISD cosourcing and multisourcing projects [57].

In discussions between IS user and supplier organizations regarding ISD project success metrics, IS suppliers may strongly advocate for the reliance on performance metrics (only). The argumentation is that money spent, time used and deliverables are objective, tangible and easily measurable [38]. Although the argument is true, reliance on ISD project performance success metrics alone has two major limitations. An agreement, especially a binding IS purchase contract with fixed time, money and deliverable objectives signed by the parties prior to the start of ISD project leads to the selection and use of plan-driven ISDMs without considering change-driven ISDMs. Secondly, ISD project performance metrics are seldom related to the business objectives and metrics of an ISD project and the business development behind the project, nor guarantee the achievement of business benefits $[6,45]$. Reliable measurement of ISD projects' business benefits is difficult due to the time delay between ISD development and its benefits realization as well as due to intervening factors, such as, changes in the inner and outer business circumstances. IS suppliers are seldom able to influence business benefits realization with their actions [45, 60]. Despite of these issues, we argue that an IS user organization needs to select and use ISDMs that support the achievement of business objectives set for IS and business development. To sum up, recent developments have driven IS user organizations towards new balances between outsourced and in-house ISD including incentives for backsourcing and insourcing. IS user organizations with insourcing, backsourcing, cosourcing and multisourcing ISD need to have sufficient understanding about ISDM and their selection. Sufficient understanding also helps them to avoid lockins and high switching costs [7,29] and to avoid management fashions [5] in ISD sourcing decisions.

\subsection{Plan-driven and Change-driven ISDMs}

Plan-driven (e.g. waterfall) and change-driven (agile) ISDMs constitute the two extremes of ISDMs [17]. All ISDMs fall between these two extremes. Plan-driven IS and other development methods assume that it is possible to plan every aspect of development work thoroughly in advance, such as, objectives and their metrics, tasks, money and resources needed. The development starts after the planning phase is completed $[49,55]$. In plan-driven methods, planning and development are typically divided into phases, such as in the waterfall method by Royce [47]. The ISD waterfall method consists of seven phases: system requirements, software requirements, analysis, program design, coding, testing and operations. Phases follow each other in a known sequential order [47]. Project management governs the execution of project phases, typically with a step-gate model. The project's steering group has to accept the results of the previous phases at a gate (=end of the phase) before the next phase is permissioned to start. Plan-driven ISDMs are seen as mechanistic and inflexible ISD methods, and critics against them have abounded for a long time [54]. 
According to Larman and Basil [34], change-driven ISDMs, or "iterative and incremental development, IID" as they call them, were used already in the early 1960's. Other well-known change-driven IID/ISDMs include prototyping and spiral models by Boehm $[8,9]$, and especially the various recent agile methods, such as, Scrum, Devops and Safe. In change-driven ISDMs, planning and development are done in small steps. Feedback from previous steps is also considered in the planning of the next step. An IS is built piece by piece guided by a generic overall plan. So called minimum viable product is typically the first IS version $[8,9,55]$. A paradigm shift is visible in the ISDM selections of the last 20 years. Plan-driven ISDMs dominated selections during the 1970's, 1980's and 1990's, whereas the popularity of change-driven ISDMs has grown during the two recent decades and appear now as the mainstream $[49,58]$. However, there is still need for plan-driven ISDMs, as well $[17,58]$.

The use of change-driven ISDMs underscores the importance of ISDM (selection) competence in a similar way as backsourcing does in a general way. In change-driven ISDMs, the tasks and participation of IS user organizations are wider and more active than in plan-driven (waterfall) ISDMs. IS user organizations are responsible for use cases, user stories, user testing and feedback and they participate into the daily ISD work. Some change-driven ISDMs actually resemble cosourcing and/or multisourcing ISD. Their use with the resulting ISD backsourcing may come as an unplanned and unwanted surprise to an IS user organization having outsourced its ISD. In a previous study [33], we discovered that IS user organizations had limited knowledge about ISDMs and the likely consequences of ISDM selection and use.

\subsection{Extant Recommendations of ISDM Selection Models Found in Literature}

With a systematic literature study [18], we found over 1000 unique scientific publications about ISDM selection. However, only 42 of them compared the selection of alternative ISDMs. Most listed ISDM selection criteria only. The number of publications with ISDM selection models was clearly lower, only 16 publications. Of them only half had original models, the rest were more or less copies and modifications of earlier models. The most complex ISDM selection model included 28 factors [3], whereas the majority of models had two or three factors [e.g. 13]. The already mentioned Boehm and Turner model [10] consisted of five factors. The most typical ISDM selection model proposed that ISDMs should be selected based on the ISD project complexity and uncertainty. Figure 1 illustrates an example [13].

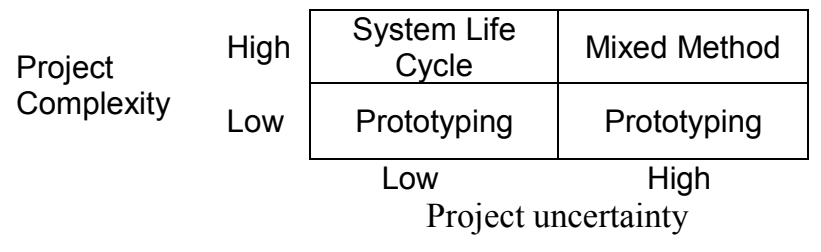

Figure 1. The ISDM selection model of Burns and Dennis (1985) (System Life Cycle = plan-driven)

We were unable to find empirical evaluations on the usefulness of ISDM selection criteria and/or models, or about the use experience and popularity of alternative ISDM selection models. The popularity of ISD outsourcing could be the reason for this. At the time when robust ISD practice and research based ISDM selection models were finally proposed, such as, the Boehm and Turner model in 2004, IS user organizations had lost their interest in ISD and ISDMs. In consequence, we could not use any of the ISDM selection models as a "baseline" for the empirical evaluations of our study. Instead of that, we calculated the frequencies of terms and concepts used in the ISDM selection criteria lists and models. We then added to that ISDM selection assumptions/propositions found in the 42 reviewed publications. By doing this, we were able to condense the knowledge about the ISDM selection recommendations, criteria lists and models in prior research into nine ISDM selection recommendations for empirical evaluation. More detailed tables on the ISDM selection criteria and model reviews are available in $[18,33]$.

The uncertainties of an ISD project outcomes is a typical selection criteria and model factor in the ISDM selection literature. Change-driven ISDMs are seen to suit better to the management of these uncertainties than plan-driven ISDMs [10, 13, 26, 32, 44, 51, 60].

Recommendation 1: High ISD project outcome uncertainties favor change-driven ISDMs.

The complexity of an ISD project is an ambiguous theoretical concept. Burns and Dennis [13] and Saarinen [48] define complexity almost as a synonym for the ISD project size. On the other hand, Mathiassen and Stage [37] asked, whether ISD project uncertainty and complexity are independent or elements of the same concept. Howell et al. [25] proposed that complexity could be regarded as one element of uncertainty. The ISD project size (complexity) is often mentioned as an ISDM selection criteria or factor. Plan-driven ISDMs are seen to suit better to large ISD projects [e.g. 10, 19, 22, 36]).

Recommendation 2: Large (complex) ISD project size favors plan-driven ISDMs.

A typical ISD outcome-related proposition in prior research is that plan-driven ISDMs deliver higher quality ISs than change-driven ISDMs. As IS quality 
appeared to us as a multi-dimensional theoretical concept, we divided this concept into three different recommendations (3-5). All three recommendations follow the formulation of the generic proposition that plan-driven ISDMs deliver higher quality ISs. The first recommendation addresses the criticality of the developed IS. Criticality is understood as the amount of potential losses materializing from the impacts of IS and ISD project defects [10]. Cockburn [14] divides possible losses into the four categories: loss of comfort, loss of discretionary money, loss of irreplaceable money and loss of life. Prior research recommends the use of plan-driven ISDMs since the assumed higher systematics is seen to ensure the better fulfillment of all ISD specifications [e.g. 3, 10, 22, 25, 53].

Recommendation 3: The need to develop an IS with high criticality favors plan-driven ISDMs.

The security of the developed IS is another IS quality dimension. The rationale of this recommendation is that the higher assumed systematics of plan-driven ISDMs makes it easier to develop secure ISs [e.g. 22, 23, 53].

Recommendation 4: The need to develop an IS with high security favors plan-driven ISDMs.

The final IS quality recommendation deals with the maintainability of IS. Prior research proposes that plandriven ISDMs produce more exhaustive documentation as well as better documented software code than change-driven ISDMs [e.g. 19, 22, 43].

Recommendation 5: The need to develop an IS with high maintainability favors plan-driven ISDMs.

Prior research includes several ISDM selection criteria and factors related to IS developers. Several authors regard the skills and experience of an IS developer team as one of the key criteria or factors in the ISDM selection [e.g. 3, 10, 22, 56]. Change-driven ISDMs are proposed to require better skilled and proficient IS developers than plan-driven ISDMs [e.g. $3,10,56]$. This proposition builds on the logic that the higher flexibility, adaptability and creativity of changedriven ISDMs require that IS developers have higher basic knowledge and skills [e.g. 3, 10, 22].

Recommendation 6: Reliance on lowly skilled IS developers favors plan-driven ISDMs, and reliance on highly skilled IS developers favors changedriven ISDMs.

Team size is another criterion / factor related to IS developers. Prior literature proposes that change-driven ISD is possible only with small IS developer teams, whereas plan-driven ISDMs should be used with large teams [e.g. 3, 10, 19, 22, 52]. Although prior research does not provide any clear definition for the small team size, IS developer teams with more than a dozen members are no longer seen as small teams $[2,10]$.
Recommendation 7: Small IS developer teams favor change-driven ISDMs and large teams favor plandriven ISDMs.

ISD project communication, especially IS designers' ability to communicate and collect feedback from business users, is regarded an essential element of change-driven ISDMs [e.g. 3, 10, 59]. Communication is seen to be closely related to IS users' commitment [52]. Dyck and Majchrzak [19] define communication as a part of social engineering practices with relation to an organization's co-operation culture [19]. Boehm and Turner also consider IS user organization culture [10].

Recommendation 8: Continuous interaction with IS user organization favors change-driven ISDMs, and the lack of interaction (needs) favors plandriven ISDMs.

The final recommendation captures several organizational characteristics of IS user organizations. Abrahamsson [2] and Jacobson [27] discovered that large organizations tend to prefer plan-driven ISDMs. Large organizations are more often rigid and prescriptive [27]. Ahimbisibwe et al. [3] proposed that if mechanistic and bureaucratic structures characterize an organization, then plan-driven ISDMs are preferable. Change-driven ISDMs are preferable in organizations with organic and flexible structures [3]. The culture factor of Boehm and Turner [10] suggests that in an IS user organization with many degrees of freedom change-driven ISDM should be favored. We combined these three organizational characteristics into one recommendation.

Recommendation 9: The large size of an IS user organization favors plan-driven ISDMs.

\section{Methodology of the Expert Interviews}

The interview data for this study was collected as a part of a larger study. We selected the personal faceto-face interview method for data collection. We wrote and maintained an interview protocol as advised by Yin [61] to guide interview planning and execution as well as data collection and analysis. We also kept a diary about the experiences of each interview. The interview method enables interactive synchronous communication, the asking of additional questions, the registering of body language and other social clues, which all help an interviewer and an interviewee to better understand each other [42]. Prior the interviews, we crafted several versions of the interview questions to reflect the findings of the systematic literature study [18]. The aim of crafting the interview questions was to have simple, direct and neutral questions with enough variation to get rich data [30]. We also followed the recommendations of Myers and Newman [40] and planned a clear interview drama. We 
conducted two rehearsal interviews and fine-tuned the interview questions, for example, added Likert scale to the nine ISDM selection recommendation questions. The fine-tuned questions were sent to four academics and two senior consultants with academic background. Another fine-tuning round was carried out to include their comments although most interview questions remained unchanged.

The objective written into the case protocol was to conduct at least 20 interviews. We, however, continued interviews until nothing new emerged, that is, until data saturation was achieved. Cumulatively 31 interviews (including the two rehearsal interviews) were conducted during the spring 2016.

ISDM consultants and professionals working on the borderline between IS suppliers and IS user organizations were recruited as interviewees. To have a "variety of voices" [40] interviewees were selected in cooperation with the Association for Information Systems Developers and the local Finnish Software Measurement Association. We also used "snowball sampling" by asking every interviewee to recommend a person who should be interviewed next. The interviewees had a long history in ISD projects with the average of 20 years' experience. They had cumulatively participated into over 1000 ISD projects, knew plan-driven and change-driven ISDMs, and, with the exception of one person, they had experience of several ISD projects with both types of ISDMs.

The interviews were semi-structured and standardized to better enable data analysis of collected data. An interview began with open ended questions about the interviewees' experiences [30]. Closed, more specific questions were placed at the end of the interview [40]. Questions about the usefulness of the nine ISDM selection recommendation was the last set of questions in the interview.

The challenges of an interview are to listen and understand the responses of the interviewee and, at the same time, ensure that all questions are answered within the time-frame reserved for the interview [42]. To tackle these challenges and to increase the reliability of the responses, we followed the interview method protocol developed by Dahlberg, Hokkanen and Newman [16]. During an interview, the questions were presented one by one on a screen to the interviewee, and the interviewer typed down the responses right away before moving to the next question. Typing down the responses did not disrupt the conversational nature of interviewing; instead, it gave interviewees more time to ponder their answers. The ISDM selection recommendation questions were also discussed and the comments were typed down even though the interviewees were asked to provide a Likert scale evaluation about each recommendation.
Two hours were reserved for each interview since typing down the responses took slightly more time than just recording responses. Interviews were also recorded. Recordings were used to verify and complement responses. The thus verified and completed interview texts were sent to the interviewees for acceptance. Out of 31 interviewees 14 responded by returning slightly modified responses and the other 17 interviewees accepted the written interview narrative without changes.

Immediate feedback from the interviewees was one of the strengths in the interview method used. As an interviewee saw all the time what was written down, (s)he was able to make corrections immediately. Both the interviewee and the interviewer saw and shared the same response text (but could still understand the meaning differently [30]). The method ensured that all interviewees verified and accepted their responses.

In our opinion, the interview method proved its usefulness in our study. We interviewed experienced ISDM experts, who wish tell "war stories". They have a lot of experience of various ISD projects, of different user and IS supplier organizations, and of several ISDMs. These facts do not, however, guarantee that they would be impartial observers. In real life projects, our interviewees follow the rules and practices of their employers. Those rules and practices could be biased to the use of particular ISDM(s). Even though we asked the interviewees to express their personal opinions and to describe their own experiences, we are unable to evaluate whether or not they behaved in this way. No documents or other sources of data were available for data triangulation. On the other hand, we were able to document why an interviewee responded the way (s)he did. The method allowed us to continue interviews until data saturation was reached since we were able to assess the saturation after each interview.

\section{Results}

We were able to collect full sets of answers from 28 interviewees. One interviewee did not have the time for this last part of the interview, and, in the two rehearsal interviews, the relevant questions were not expressed on the Likert scale.

The key findings are presented in Table 1. It shows the weights to each response alternative (agree strongly, moderately, slightly, or is neutral, or disagree slightly, moderately or strongly) and the distribution of the responses. The last columns display the weighted sums of scores for each recommendation with 28 as the theoretical minimum and 198 as the maximum and other descriptive statistics.

Table 1 discloses large variations between the scores of recommendations and also in the distribution 
of answers to most recommendations. The only recommendation receiving strong support was the first recommendation; high ISD project outcome uncertainties favor change-driven ISDMs. The average of responses was 6; strongly agree. The sixth recommendation about the skills of IS developers received moderate support with a 4.9, moderately

1. ISDM experts' evaluations about the usefulness of recommendations in ISDM selection models

\begin{tabular}{|c|c|c|c|c|c|c|c|c|c|c|}
\hline Recommendation & 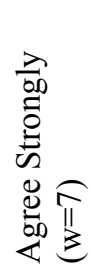 & 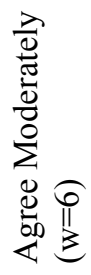 & 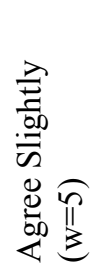 & 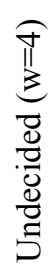 & 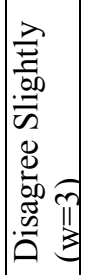 & 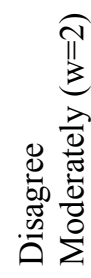 & 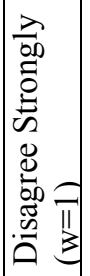 & 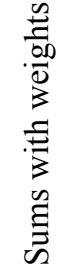 & 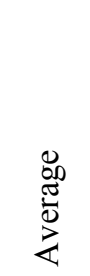 & 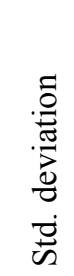 \\
\hline $\begin{array}{l}\text { Recommendation 1: High ISD project outcome } \\
\text { uncertainties favor change-driven ISDMs. }\end{array}$ & 14 & 12 & 2 & 0 & 0 & 0 & 0 & 180 & 6,43 & 0,63 \\
\hline $\begin{array}{l}\text { Recommendation 2: Large (complex) ISD project } \\
\text { size favors plan-driven ISDMs. }\end{array}$ & 1 & 4 & 9 & 0 & 3 & 7 & 4 & 103 & 3,68 & 1,91 \\
\hline $\begin{array}{l}\text { Recommendation 3: The need to develop an IS with } \\
\text { high criticality favors plan-driven ISDMs. }\end{array}$ & 0 & 7 & 4 & 0 & 5 & 6 & 6 & 95 & 3,39 & 1,97 \\
\hline $\begin{array}{l}\text { Recommendation 4: The need to develop an IS with } \\
\text { high security favors plan-driven ISDMs. }\end{array}$ & 0 & 2 & 5 & 3 & 6 & 7 & 5 & 86 & 3,07 & 1,59 \\
\hline $\begin{array}{l}\text { Recommendation 5: The need to develop an IS with } \\
\text { high maintainability favors plan-driven ISDMs. }\end{array}$ & 0 & 1 & 5 & 1 & 4 & 10 & 7 & 74 & 2,64 & 1,54 \\
\hline $\begin{array}{l}\text { Recommendation 6: Reliance on lowly skilled IS } \\
\text { developers favors plan-driven ISDMs, and ... }\end{array}$ & 7 & 9 & 2 & 2 & 2 & 5 & 1 & 138 & 4,93 & 2 \\
\hline $\begin{array}{l}\text { Recommendation 7: Small IS developer teams favor } \\
\text { change-driven ISDMs and ... }\end{array}$ & 2 & 6 & 5 & 0 & 2 & 9 & 4 & 103 & 3,68 & 2,09 \\
\hline $\begin{array}{l}\text { Recommendation 8: Continuous interaction with IS } \\
\text { user organization favors change-driven ISDMs, ... }\end{array}$ & 1 & 8 & 5 & 1 & 7 & 4 & 2 & 115 & 4,11 & 1,79 \\
\hline $\begin{array}{l}\text { Recommendation 9: The large size of an IS user } \\
\text { organization favors plan-driven ISDMs }\end{array}$ & 0 & 1 & 5 & 0 & 6 & 10 & 6 & 75 & 2,68 & 1,49 \\
\hline
\end{tabular}

As a whole, the interviewed ISDM experts perceived the usefulness of the ISDM selection frameworks' recommendations low and disagreed with most recommendations. The averages of responses to recommendations 5 (IS maintainability favors plan-driven ISDMs) and 9 (large size of organizations favors plan-driven ISDMs) were 2.7, which is close to the middle of disagree strongly and disagree slightly. Also, the averages of the remaining recommendations were on the disagree-side with averages between 3.1 and 3,7, and with the exception of recommendations 8 with a 4.1 average.

When discussing the high maintainability need of ISs, several interviewees explained that high IS maintainability is possible to achieve both with plandriven and change-driven ISDMs. One interviewee stated: "No method forces you do poor quality work". The interviewees stressed the prioritization of ISD objectives in their evaluations of recommendations 3 , 4 and 5 (the three dimensions of IS quality). The tone agree, average. One interviewee agreed fully with the sixth recommendation and stated: "someone [skilled enough] is required to produce a plan". Another interviewee disagreed with the sixth recommendation and explained that good skills are needed also with plan-driven ISDMs: "With poor skills you should not start at all". 
developer team) was rather similar. Some interviewees agreed with these recommendations, whereas other interviewees explained that large and complex ISD projects and large IS developer teams are always a problem. A few interviewees claimed that with the Safe (Scaled Agile Framework) ISDM, change-driven ISD could be scaled to large IS development teams and complex ISD projects.

The recommendation number eight (8) produced the widest distribution of opinions. Those who disagreed with the recommendation commented that the lack of interaction and communication will eventually create problems. One interviewee coined this: "Regardless of the method, there will be problems".

A few interviewees saw little value in plan-driven ISDMs and favored strongly change-driven ISDMs. One of them encapsulated this: "You have to plan, but it doesn't mean that you should use plan-driven ISDMs". Most interviewees discussed hybrid methods, i.e., the combination of plan-driven and change-driven ISDMs, even though there was no interview question about hybrid methods. In summary, based on the ISDM expert interviews, the usefulness of investigated extant ISDM selection recommendations were perceived low and the findings of ISDM selection research outdated. A good question is: how to augment the usefulness of ISDM selection models and their recommendations.

\section{Discussion and Conclusions}

In this article, we condensed the knowledge of ISDM selection criteria lists, ISDM selection model factors and the related ISDM selection propositions into nine well-defined ISDM selection recommendations. None of these recommendations addressed the characteristics of business development. In our opinion, this a serious limitation in the ISDM selection research, especially in contexts where ISD is closely linked to business development. The purpose of ISD work is to support business development. This is our answer to the two research question outlined in the Introduction Section.

In our opinion, these nine recommendations capture well the extant knowledge base of the ISDM selection research. It is, however, necessary to point out that the ISDM selection research has used various theories and theoretical concepts and combined them with practical ISD experiences. It is also necessary to point out that we were unable to detect a dominant ISDM selection criteria list or an ISDM selection model although we regard the model of Boehm and Turner [10] the best-known model. Due to this situation, it is possible that we have made mistakes in condensing the knowledge into the nine recommendations. Nevertheless, we have done our best to show how the recommendations were created and condensed from prior research.

We asked 28 ISDM experts to evaluate the usefulness of the nine ISDM selection recommendations on the Likert scale and also express verbally what they thought about the recommendations. With the exception of two recommendations, the interviewees perceived the usefulness of literature based ISDM selection recommendation low. According to these two highly evaluated recommendations, plan-driven ISDMs should be used when the competences of IS developers are low, and change-driven ISDMs when the uncertainties of ISD project outcomes are high and/or the competences of IS developers are high. This is our response to the second research question.

It was a surprise to us that the ISDM experts were so critical towards the extant ISDM selection recommendations of prior research. Based on this, it appears to us that both the findings of ISDM selection research and the related knowledge base are severely outdated. The development and maturing of change-driven ISDMs during the recent years and the changes in the role of IS, ISD and ISDMs appear as potential reasons behind this finding. Consequently, there is a clear need for enhanced ISDM selection models and ISDM selection recommendations.

We propose that the usefulness of ISDM selection models and related recommendations can best be augmented by combining the characteristics of IS and business development. This proposition means that the characteristics of ISDM(s) selected and used need to match with the characteristics of the business development methods selected and used and vice versa. This is an amenable venue of future research.

Based on the systematic literature study and the ISDM expert interviews, we have conducted also other studies. In one prior study [18], we proposed a new ISDM selection framework that matches the characteristics of ISDMs with the characteristics of business development contexts. In doing so, we combined the theoretical basis of ISDM research with business development and organization theory research. We also interviewed the 31 ISDM experts about the dimensions and the factors of the new ISDM selection model and about the usefulness of the approach and the entire framework. Of the 31 interviewees, 28 supported partially or fully the usefulness claim of the approach and the framework whereas three experts disagreed. The development of ISDM recommendations from this framework or similar studies is another possible venue for future 
research. The present research could also be repeated in organizations where ISD is insourced.

The proliferation of ISD backsourcing, cosourcing and multisourcing were discussed in the two first Sections of this article. Our advice to practitioners, especially to IS user organizations, is to ensure in these cases that they acquire enough competences so that they are able to understand sufficiently the possibilities and limitations of the most typical ISDMs to make ISDM decisions from business benefits perspective and to execute plandriven and/or change-driven ISD projects.

ISDM selection has been a neglected research area during the past 15 years. Our advice to researchers is to investigate ISDMs again, and not only as an ISD issue, but as a business development issue. With our article, we contribute to this research by condensing the knowledge of ISDM selection research into well-defined recommendations, by probing the perceived usefulness of these recommendations empirically, and by suggesting how to augment them.

\section{References}

[1] Abrahamson, E. Management Fashion. The Academy of Management Review 21, 1 (1996), 254-285.

[2] Abrahamsson, P., Salo, O., Ronkainen, J., and Warsta, J. Agile software development methods. VTT Publications VTT Publ., 478 (2002), 112.

[3] Ahimbisibwe, A., Cavana, R.Y., and Daellenbach, U. A contingency fit model of critical success factors for software development projects A comparison of agile and traditional plan-based methodologies. Journal of Enterprise Information Management 28, 1 (2015), 7-33.

[4] Alaghehband, F.K., Rivard, S., Wu, S., and Goyette, S. An assessment of the use of Transaction Cost Theory in information technology outsourcing. Journal of Strategic Information Systems 20, 2 (2011), 125-138.

[5] Alvesson, M. and Spicer, A. A Stupidity-Based Theory of Organizations. Journal of Management Studies 49, 7 (2012), 1194-1220.

[6] Atkinson, R. Project management: cost time and quality two best guesses and a phenomenon, it's time to accept other success criteria. International Journal of Project Management 17, 6 (1999), 337-342.

[7] Von Bary, B. and Westner, M. Information Systems Backsourcing: a Literature Review. Journal of Information Technology Management XXIX, 1 (2018), 78.

[8] Boehm, B.W. Spiral Model of Software Development and Enhancement. Computer 21, 1988, 61-72.

[9] Boehm, B.W., Gray, T.E., and Seewaldt, T. Prototyping Vs. Specifying. IEEE Software, May (1984), 473-484.

[10] Boehm, B.W. and Turner, R. Balancing agility and discipline: A guide for the perplexed. Addison-Wesley Professional, 2004.

[11] Borg, M., Olsson, T., Franke, U., and Assar, S. Digitalization of Swedish Government Agencies - A
Perspective Through the Lens of a Software Development Census. International Conference on Software

Engineering, (2018).

[12] Brooks, F.P.J. No silver bullet - essence and accidents of software engineering. Proceedings of the IFIP Tenth World Computing Conference, (1986), 1069-1076.

[13] Burns, R.N. and Dennis, A.R. Selecting the appropriate application development methodology. $A C M$ Sigmis Database, 613 (1985).

[14] Cockburn, A. Selecting a project's methodology. IEEE Software 17, 4 (2000), 64-71.

[15] Cusumano, M.A., MacCormack, A., Kemerer, C.F., and Crandall, W. Critical Decisions in Software

Development: Updating the State of the Practice. IEEE Software 26, 5 (2009), 84-87.

[16] Dahlberg, T., Hokkanen, P., and Newman, M. How Business Strategy and Changes to Business Strategy Impact the Role and the Tasks of CIOs: An Evolutionary Model. Proceedings of the Annual Hawaii International Conference on System Sciences, (2016), 4910-4919. [17] Dahlberg, T. and Lagstedt, A. There Is Still No " Fit for All " IS Development Method : Business Development Context and IS Development Characteristics Need to Match. Proceedings of the 51st Hawaii International Conference on System Sciences, (2018).

[18] Dahlberg, T. and Lagstedt, A. A Contingency Theory Motivated Framework to Select Information System Development Methods. Pacific Asia Conference on Information Systems, (2018), 1-14.

[19] Dyck, S. and Majchrzak, T.A. Identifying common characteristics in fundamental, integrated, and agile software development methodologies. Proceedings of the Annual Hawaii International Conference on System Sciences, (2011), 5299-5308.

[20] Ebert, C., Kuhrmann, M., and Prikladnicki, R. Global software engineering: Evolution and trends. Proceedings 11th IEEE International Conference on Global Software Engineering, ICGSE 2016, (2016), 144-153.

[21] Fuggetta, A. and Di Nitto, E. Software Process. Proceedings of the on Future of Software Engineering FOSE 2014, (2014), 1-12.

[22] Guntamukkala, V.., Wen, H.J.., and Tarn, J.M.. An empirical study of selecting software development life cycle models. Human Systems Management 25, 4 (2006), 265-278.

[23] Gupta, D. and Dwivedi, R. a Framework To Support Evaluation of Project in-Hand and Selection of Software Development Method. Journal of Theoretical and Applied Information Technology 73, 1 (2015), 137-149.

[24] Hall, J.G. and Rapanotti, L. Towards a DesignTheoretic Characterisation of Software Development Process Models. GTSE '15: Proceedings of the Fourth SEMAT Workshop on General Theory of Software Engineering, IEEE Press 2015 (2015), 3-14.

[25] Howell, D., Windahl, C., and Seidel, R. A project contingency framework based on uncertainty and its consequences. International Journal of Project Management 28, 3 (2010), 256-264.

[26] Ika, L.A. Project success as a topic in project management journals. Project Management Journal 40, 4 (2009), 6-19. 
[27] Jacobson, B.I., Spence, I., and Ng, P. Agile and SEMAT - Perfect Partners. Communications of the ACM 56, 11 (2013), 53-60.

[28] Johansson, B., Hallén, O., Persson, A., and Tarland, R. Sourcing Trends Predictions - An Analysis of Regions and Strategies. Procedia Computer Science 121, (2017), 716723.

[29] Kaiser, K.M. and Hawk, S. Evolution of Offshore Software Development: from Outsourcing to Cosourcing. MIS Quarterly Executive 3, 2 (2004), 69-81.

[30] Kaplan, B. and Maxwell, J.A. Qualitative research methods for evaluating computer information systems. In Evaluating the organizational impact of healthcare information systems. Springer, New York, 2005, 30-56. [31] Kettunen, P. and Laanti, M. How to steer an embedded software project: Tactics for selecting the software process model. Information and Software Technology 47, 9 (2005), 587-608.

[32] Lacity, M.C., Khan, S.A., and Willcocks, L.P. A review of the IT outsourcing literature: Insights for practice. Journal of Strategic Information Systems 18, 3 (2009), 130-146.

[33] Lagstedt, A. and Dahlberg, T. Understanding the Rarity of ISD Method Selection - Bounded Rationality and Functional Stupidity. Pacific Asia Conference on Information Systems, (2018), 1-14.

[34] Larman, C. and Basili, V.R. Iterative and incremental development: A brief history. Computer 36, 6 (2003), 4756.

[35] Madsen, D.Ø. Examining the popularity trajectory of outsourcing as a management concept. Problems and Perspectives in Management 15, 2 (2017), 178-195. [36] Mahmood, M. a. System development methods - A comparative investigation. MIS Quarterly: Management Information Systems 11, 3 (1987), 293-307.

[37] Mathiassen, L. and Stage, J. Complexity and uncertainty in software design. COMPEURO'90:

Proceedings of the 1990 IEEE International Conference on Computer Systems and Software Engineering@m_Systems Engineering Aspects of Complex Computerized Systems, (1990), 482-489.

[38] McLeod, L., Doolin, B., and MacDonell, S.G. A perspective-based understanding of project success. Project Management Journal 43, 5 (2012), 68-86.

[39] Moe, N.B., Aurum, A., and Dybå, T. Challenges of shared decision-making: A multiple case study of agile software development. Information and Software Technology 54, 8 (2012), 853-865.

[40] Myers, M.D. and Newman, M. The qualitative interview in IS research: Examining the craft. Information and Organization 17, 1 (2007), 2-26.

[41] Nyrhinen, M. and Dahlberg, T. Is Transaction Cost Economics Theory Able to Explain Contracts Used for and Success of Firm-wide IT-Infrastructure Outsourcing? Proceedings of the 40th Hawaii International Conference on System Sciences (HICSS'07), (2007), 1-10.

[42] Opdenakker, R. Advantages and Disadvantages of Four Interview Techniques in Qualitative Research. Forum Qualitative Sozialforschung / Forum: Qualitative Social Research 7, 2006. http://nbn-resolving.de/urn:nbn:de:0114fqs0604118.
[43] Palvia, P. and Nosek, J.T. A field examination of system life cycle techniques and methodologies. Information \& Management 25, 2 (1993), 73-84. [44] Petter, S., DeLone, W., and McLean, E.R. The Past, Present, and Future of "IS Success." Journal of the Association for Informaiton Systems 13, (2012), 341-362. [45] Pinto, J.K. and Slevin, D.P. Project success: Definition and Measurement Techniques. Project Management Journal 19, 3 (1988), 67-73.

[46] Ross, J.W., Weill, P., and Robertson, D.C. Enterprise Architecture as Strategy. Harvard Business School Press, Boston, Massachusetts, 2006.

[47] Royce, D.W.W. Managing the Development of large Software Systems. Ieee Wescon, August (1970), 1-9. [48] Saarinen, T. System development methodology and project success An assesment of situational approaches. Information \& Management 19, 1 (1990), 183-193. [49] Salo, O. Enabling Software Process Improvement in Agile Software Development Teams and Organisations. $V t t$ Publications, (2006), 153.

[50] Sauer, C. and Lau, C. Trying to adopt systems development methodologies - a case-based exploration of business users' interests. Information Systems Journal 7, 4 (1997), 255-275.

[51] Savolainen, P., Ahonen, J.J., and Richardson, I. When did your project start? - The software supplier's perspective. Journal of Systems and Software 104, (2015), $32-40$.

[52] Sharon, I., Dos Santos Soares, M., Barjis, J., Van Den Berg, J., and Vrancken, J. A decision framework for selecting a suitable software development process. ICEIS 2010 - Proceedings of the 12th International Conference on Enterprise Information Systems 3 ISAS, November 2015 (2010), 34-43.

[53] Siddique, L. and Hussein, B. a. Practical Insight About Choice of Methodology in Large Complex Software Projects in Norway. Technology Management Conference (ITMC), 2014 IEEE International., (2014), 0-3.

[54] Sommerville, I. Software Process Models. ACM Computing Surveys 28, 1 (1996), 269-271.

[55] Sommerville, I. Software Engineering. AddisonWesley, Boston, Massachusetts, 2011.

[56] Tang, A. and van Vliet, H. Design Strategy and Software Design Effectiveness. IEEE Software 29, 1 (2012), 51-55.

[57] Taylor, H. Outsourced IT Projects from the Vendor Perspective: Different Goals, Different Risks. Journal of Global Information Management 15, 2 (2007), 1-27.

[58] Theocharis, G., Kuhrmann, M., Münch, J., and Diebold, P. Is water-scrum-fall reality? On the use of agile and traditional development practices. International Conference on Product-Focused Software Process Improvement, (2015), 149-166.

[59] De Weger, M.K. and Franken, H.M. A situational approach to design strategies. Software Quality Journal 6, 3 (1997), 181-194.

[60] de Wit, A. Measurement of project success. International Journal of Project Management 6, 3 (1988), 164-170.

[61] Yin, R.K. Case Study Research: Design and Methods. Sage Publications, 2009. 